\title{
Two-layer RF MEMS fractal capacitors in PolyMUMPS for S-band applications
}

\author{
Amro M. Elshurafa and Khaled N. Salama
}

Electrical Engineering, Physical Sciences and Engineering Division, King Abdullah University of Science and Technology (KAUST), Thuwal, Saudi Arabia, 23955-6900.

\begin{abstract}
In this letter, we fabricate for the first time MEMS fractal capacitors possessing two layers and compare their performance characteristics with the conventional parallel-plate capacitor and previously reported state-of-the-art singlelayer MEMS fractal capacitors. Explicitly, a capacitor with a woven structure and another with an interleaved configuration were fabricated in the standard PolyMUMPS surface micromachining process and tested at S-band frequencies. The self-resonant frequencies of the fabricated capacitors were close to $10 \mathrm{GHz}$, which is better than that of the parallel-plate capacitor which measured only $5.5 \mathrm{GHz}$. Further, the presented capacitors provided a higher capacitance when compared to the state-ofthe-art reported MEMS fractal capacitors created using a single layer at the expense of a lower quality factor.
\end{abstract}

\section{Introduction}

Radio frequency microelectromechanical (RF MEMS) circuits and devices have been receiving research attention and progressing steadily during the past 15 years because of many desirable characteristics including for example high quality factors $(Q)$ [1], low insertion losses [2], virtually non-existent power losses [3], and their strong potential in further miniaturization of wireless devices by replacing bulky off-chip components [4]. Very recently, RF MEMS capacitors utilizing fractal geometries have been fabricated and tested [5], and it was shown that the fractal 
concept provides a number of benefits that significantly enhance the performance of RF MEMS capacitors.

Fractal capacitors were introduced initially in the complementary metal oxide semiconductor (CMOS) process by the authors in [6], in which they specifically utilized the Koch Island fractal configuration, while the authors in [5] created MEMS fractal capacitors using Moore's geometry within a single layer only. In this letter, we create for the first time MEMS fractal capacitors in two layers and present a detailed analysis on the woven structure (WS) and the interleaved structure (IS) in particular. Further, the performance of these capacitors is compared to the conventional parallelplate capacitor structure (PPS) and the state-of-the-art fractal MEMS capacitors as well.

\section{Device description and fabrication}

Both the woven and interleaved structures are considered fractal geometries. Further, many of the benefits that were detailed in [5] apply to these structures including eliminating the need for etching holes, eliminating the residual stress warping, and reducing the parasitic effects.

Conceptual schematics of the woven and interleaved capacitors are shown in Fig. 1a and Fig. 1b respectively. As can be seen, both structures require two layers. Further, there are two terminals within each layer to benefit from the vertical and horizontal capacitances simultaneously.

Based on the previous elaboration, the WS and the IS were fabricated in the PolyMUMPS process (www.memscap.com), where the top layer was created using Metal+Poly2, whereas the bottom layer was created using Poly1; there is a sacrificial layer of oxide which is $0.75 \mu \mathrm{m}$ thick separating both layers. Scanning electron 
microphotographs (SEM) are provided for the WS and the IS capacitors in Fig. 2 and Fig. 3 respectively. A third capacitor was also fabricated and possessed a PPS in the same fabrication run, but an SEM is not included herein in the interest of brevity because it is well-known. All three fabricated capacitors were of the same size to ensure a fair comparison.

Because both the interleaved and woven structures contain long segments $(\sim 250 \mu \mathrm{m})$ that are very close to each other, proximity effects and polysilicon reflectivity during fabrication makes creating these structures, without compromising the lithography quality on the rest of the wafer, a difficult task as explicitly stated in the PolyMUMPS design handbook [7]. Since the minimum allowable separation in PolyMUMPS is $2 \mu \mathrm{m}$, we relax this condition by making the separation between any two adjacent segments $3 \mu \mathrm{m}$.

\section{Measurements and Discussion}

To extract the capacitance and $Q$ of the fabricated capacitors, the measurements were performed using an Agilent 8363C network analyzer and a ground-signal-ground coplanar probe from Cascade. Further, the measurements were all one-port type measurements (i.e. $S_{11}$ or return loss), and were preceded by a shortopen-load calibration procedure.

The return loss of the woven capacitor is shown in Fig. 4a, while the return loss of the interleaved capacitor is shown in Fig. 4b. The measurements in both capacitors spanned the frequency band from $1 \mathrm{GHz}$ to $4 \mathrm{GHz}$. We do not include results beyond $4 \mathrm{GHz}$ because the $Q$ after that becomes too low $($ i.e. $<4)$ to be used in any real application. The performance of all the capacitors, i.e. the WS, IS, PPS and 
single-layer fractals [5] are plotted in Fig. 5 and Fig. 6, where Fig. 5 plots the measured capacitance and Fig. 6 plots the measured $Q$.

From Fig. 5 and Fig. 6, we deduce that the PPS capacitor is the capacitor that provides the highest capacitance. However, this extra capacitance is caused by the residual stress warping [8] and also from the large parasitic capacitance created between the bottom plate and the substrate. On the other hand, most of the capacitance of the WS and IS capacitors are from the capacitor itself with little parasitic contribution $[5,6]$. The latter statement is confirmed by the self-resonant frequencies of the capacitors, which measured $5.5 \mathrm{GHz}$ for the PPS capacitor and approximately $10 \mathrm{GHz}$ for the WS and IS capacitors. The $Q$ of the woven capacitor is relatively low due to the numerous vias, while the $Q$ of the interleaved capacitor is even lower because of the steps present in the metal depositions since no chemical mechanical polishing is performed in PolyMUMPS. These steps, which could be observed in Fig. 3 at the left and the right of the IS capacitor, make the metal thinner (at the steps itself) and hence increase the series resistance.

Compared to the state-of-the-art MEMS fractal capacitors presented in [5], the capacitance acquired herein from the WS and IS capacitor is higher but $Q$ is lower. These results are indeed logical because the authors in [5] create both terminals of the capacitor in a single metal layer. The use of a single metal layer enhances $Q$ but also results in capacitances being present horizontally only, as opposed to the capacitors herein where both horizontal and vertical capacitances are present. Table 1 summarizes the performance of all capacitors in a compact manner. We note that no capacitor possesses simultaneously a high $Q$, high capacitance and a high SRF; a compromise always exists. 
Table 1: Comparative study at $2 \mathrm{GHz}$.

\begin{tabular}{|c|c|c|c|c|}
\hline Criterion & Parallel-Plate & Woven & Interleaved & Moore's \\
\hline $\boldsymbol{C}(\mathbf{p F})$ & 4.7 & 1.1 & 1.2 & 0.58 \\
\hline $\boldsymbol{Q}$ & 7 & 6 & 3.5 & 10 \\
\hline SRF (GHz) & 5.5 & 10 & 10 & 20 \\
\hline
\end{tabular}

The fabricated capacitors could have been fabricated in a CMOS process with both plates/terminals being made out of metal, and that would enhance $Q$. However, we draw the attention of the reader that among the objectives of using fractals in MEMS capacitors is to solve and/or overcome the fabrication problems that are usually encountered in MEMS processes, and MEMS process mostly possess a single metal layer among several other polysilicon layers. In other words, using a metal material for both terminals in this paper defeats the purpose of testing these capacitors for MEMS purposes.

\section{Conclusion}

The main contribution of this letter is fabricating and testing, for the first time, MEMS fractal capacitors in two layers as opposed to previously reported MEMS fractal capacitors that were created in a single layer. It was found that the interleaved and woven structures provide a lower capacitance when compared to the parallel-plate structure, but possess a significantly higher resonant frequency. On the other hand, and compared to the state-of-the-art RF MEMS fractal capacitors reported in the literature, they possess a higher capacitance at the cost of a lower quality factor. 


\section{Acknowledgments}

The authors would like to thank the personnel of the Advanced Nanofabrication, Imaging, and Characterization Core Laboratories at King Abdullah University of Science and Technology (KAUST) for their assistance in measurements and imaging especially Mr. X. Yu, Dr. M. Hedhili, Dr. A. Behzad, and Dr. C. Zhao.

\section{References}

[1] Li L., Uttamchandani D.: 'Monolithic RF MEMS inductor using silicon MEMS foundry process', IET Micro Nano Letters, 2006, 1, pp. 5-8.

[2] Li L., Uttamchandani D.: 'MEMS microwave device with switchable capacitive and inductive states', IET Micro Nano Letters, 2008, 3, pp. 77-81.

[3] Ghodsian B., Bogdanoff P., Hyman D.: 'Wideband DC-contact MEMS series switch', IET Micro Nano Letters, 2008, 3, pp. 66-69.

[4] Nguyen C.T.C.: 'MEMS technology for timing and frequency control', IEEE Trans. Ultrasonics, Ferroelectrics and Freq. Control, 2007, 54, pp. 251-270.

[5] Elshurafa A.M., Radwan A.G., Emira A., Salama K.N.: 'RF MEMS Fractal Capacitors With High Self-Resonant Frequencies', IEEE J. Microelectromech. Syst., 2012, 21, pp. 10-12.

[6] Samavati H., Hajimiri A., Shahani A., Nasserbakht G., Lee T.: 'Fractal capacitors', IEEE J. Solid-State Circuits, 1998, 33, pp. 2035-2041.

[7] J. Carter, et al.: 'PolyMUMPS Design Handbook - Rev. 11', MEMSCAP Inc., Bernin, France.

[8] Elshurafa A.M., El-Masry E.: 'The effects of non-uniform nanoscale deflections on capacitance in RF MEMS parallel-plate variable capacitors', $J$. Micromech. Microeng., 2008, 18, article id 045012. 


\section{Figure captions}

Fig. 1. Conceptual schematics of two-layer fractal capacitors

$a$ The woven structure comprising a bottom layer, a top layer and vias to connect the segments with the same polarity

$b$ The interleaved layer comprising a top layer and a bottom layer. Note that the polarity is interchanging in both structures.

Fig. 2. The fabricated woven capacitor in the PolyMUMPS process. Notice the measurement pads on the left.

Fig. 3. The fabricated interleaved capacitor in the PolyMUMPS process. Notice the measurement pads to the left.

Fig. 4. Measurement results

$a$ The $S_{11}$ measurements for the woven capacitor

$b$ The $S_{11}$ measurements for the interleaved capacitor

Fig. 5. Measured capacitance versus frequency with Moore's measurements from [5].

Fig. 6. Measured quality factor versus frequency with Moore's measurements from [5]. 


\section{Figures}

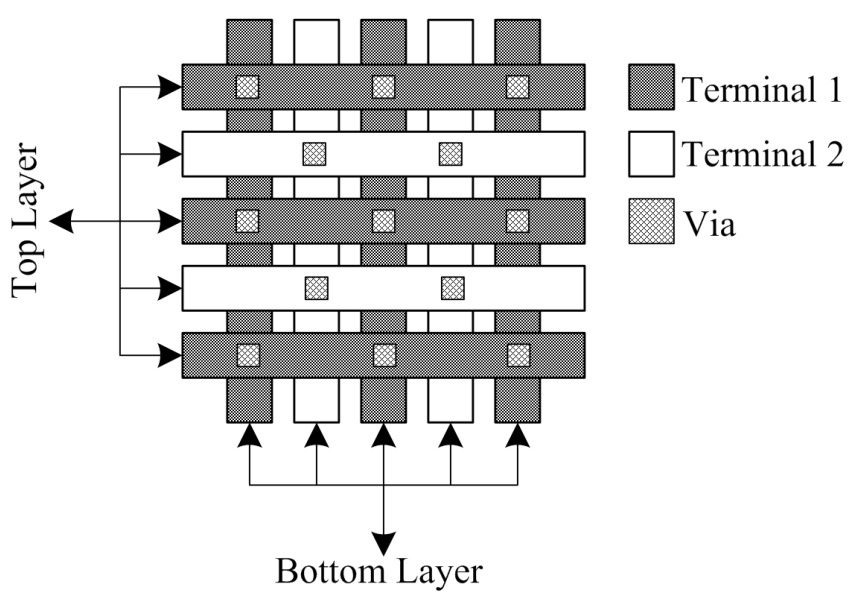

$a$

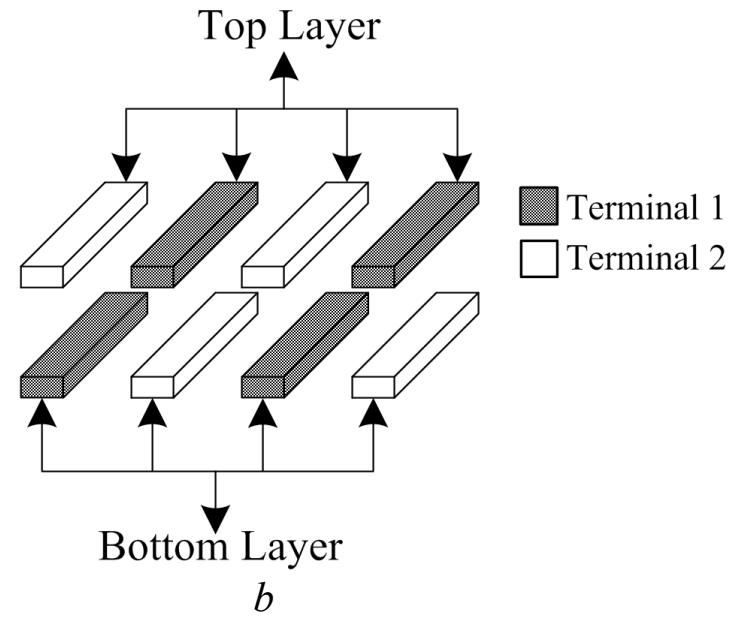

Fig. 1. Conceptual schematics of two-layer fractal capacitors

$a$ The woven structure comprising a bottom layer, a top layer and vias to connect the segments with the same polarity

$b$ The interleaved layer comprising a top layer and a bottom layer. Note that the polarity is interchanging in both structures. 


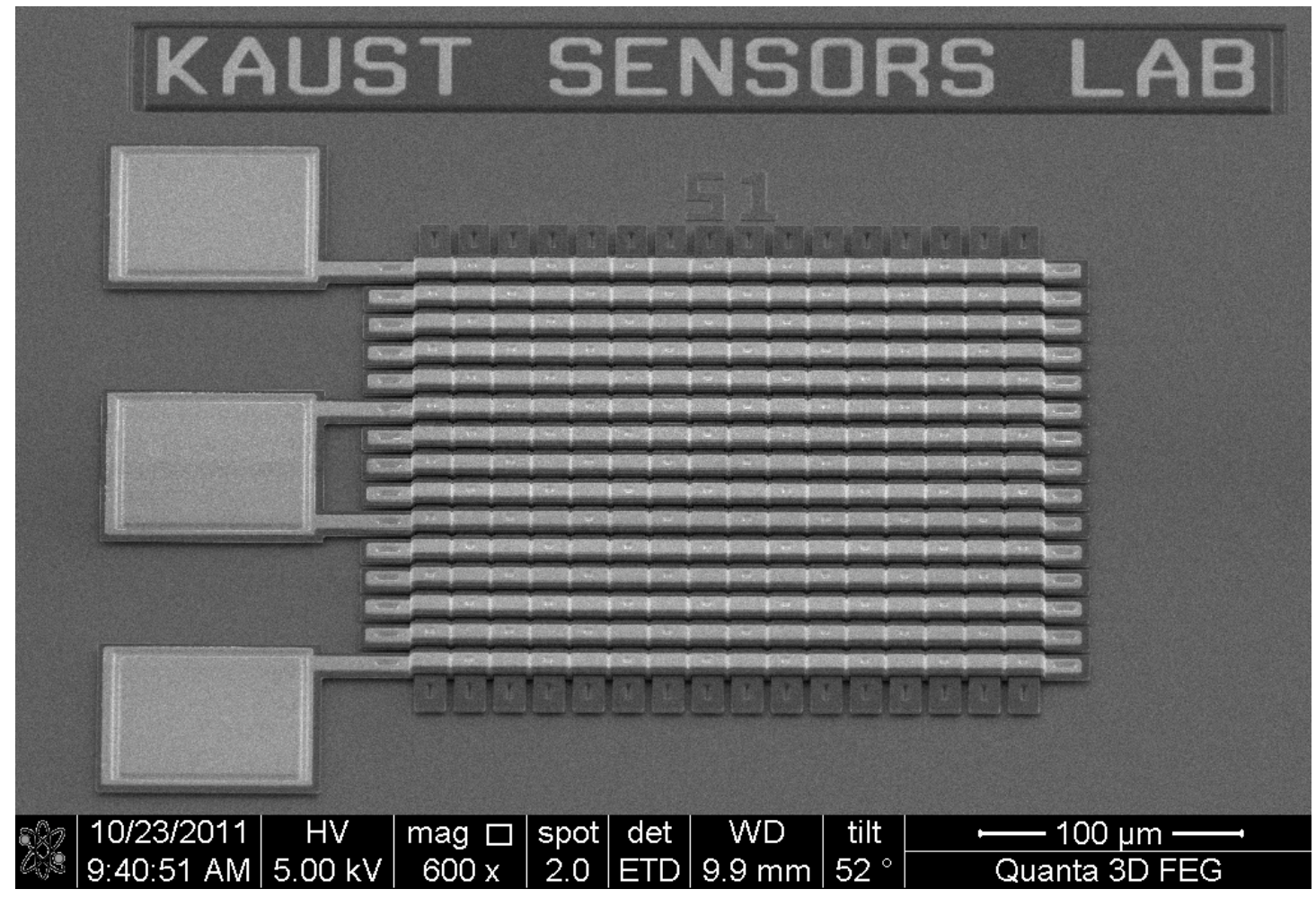

Fig. 2. The fabricated woven capacitor in the PolyMUMPS process. Notice the measurement pads on the left. 


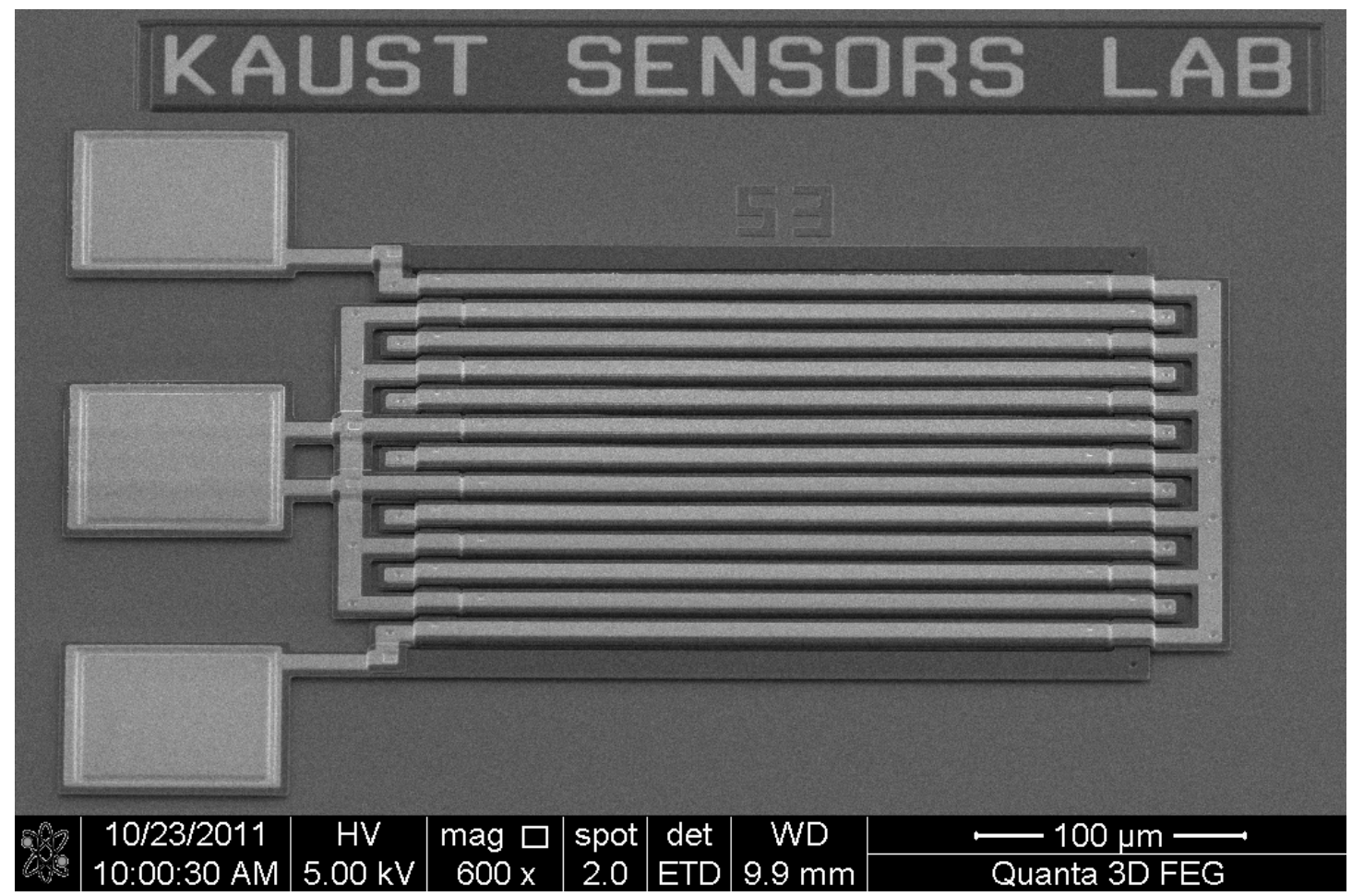

Fig. 3. The fabricated interleaved capacitor in the PolyMUMPS process. Notice the measurement pads to the left. 


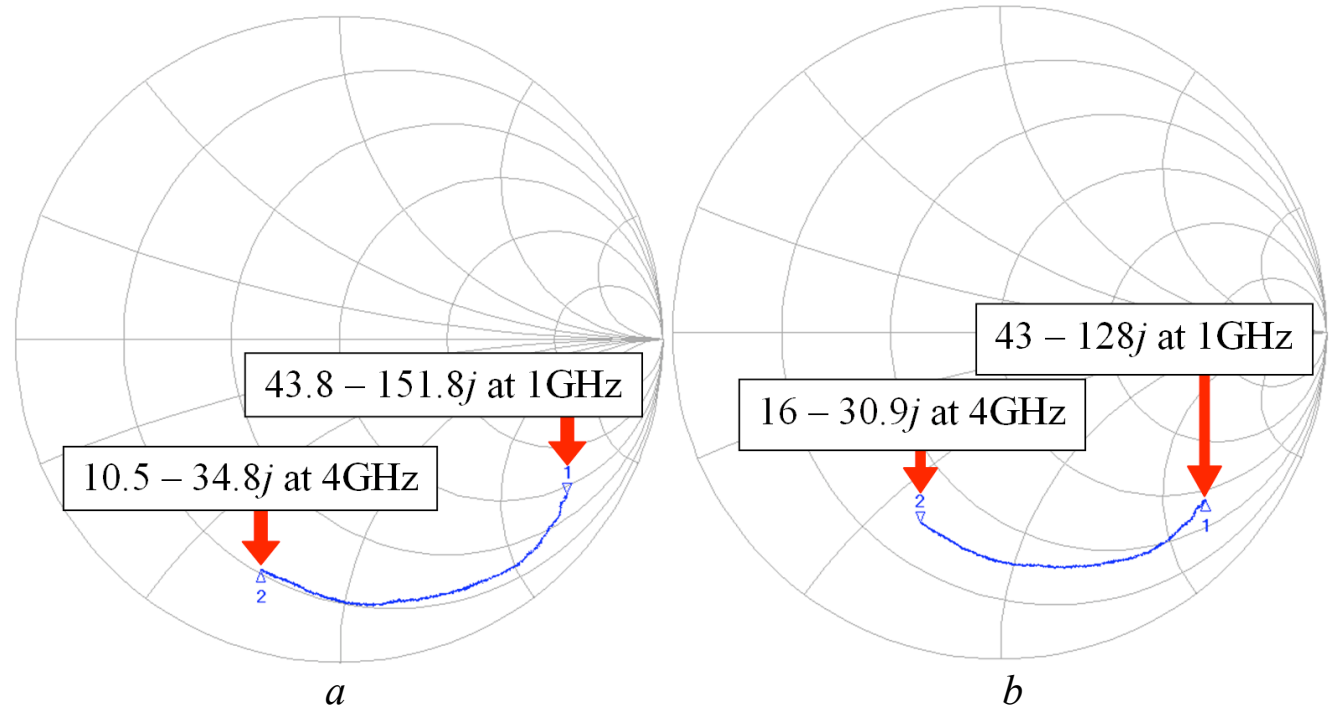

Fig. 4. Measurement results

$a$ The $S_{11}$ measurements for the woven capacitor

$b$ The $S_{11}$ measurements for the interleaved capacitor 


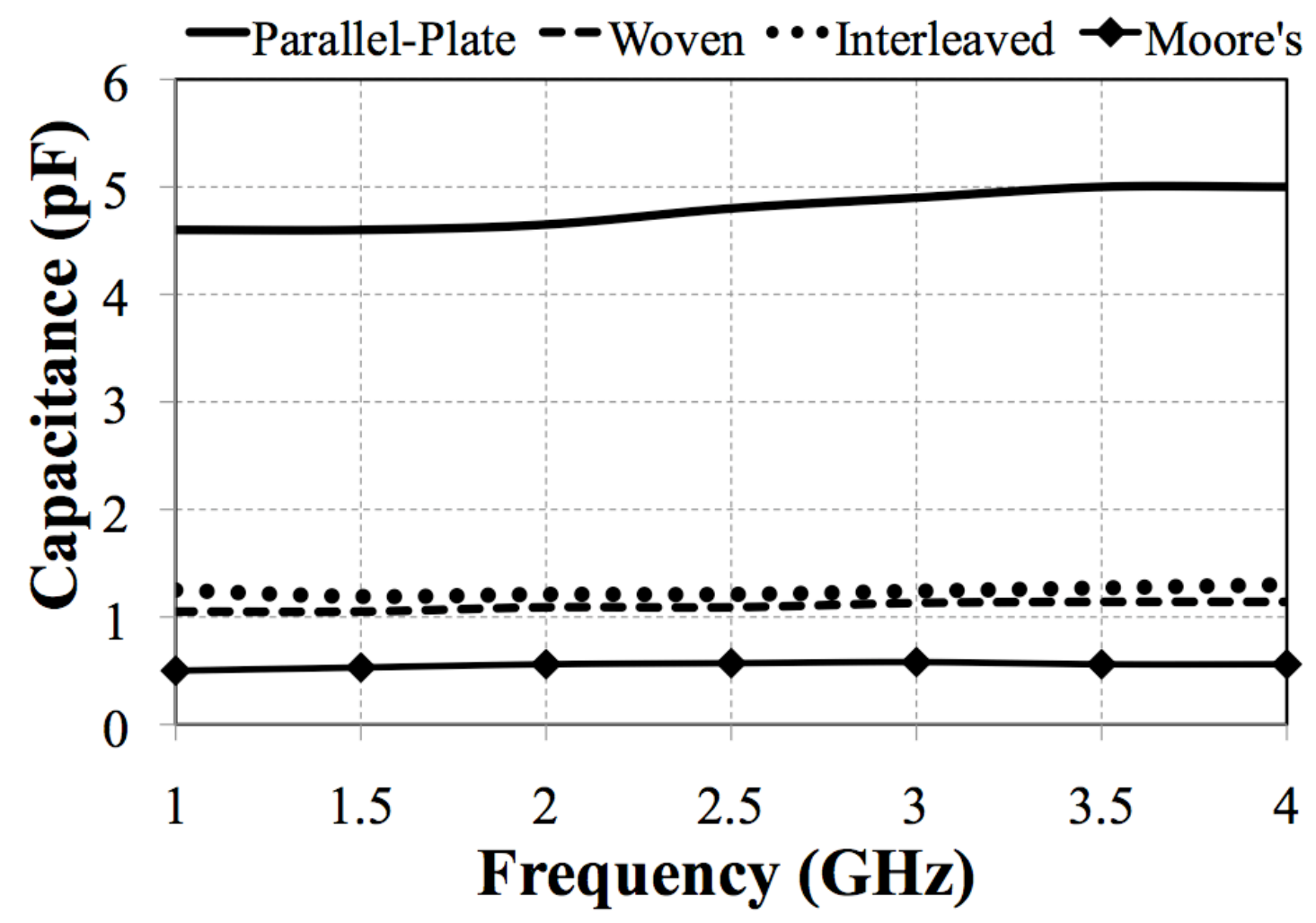

Fig. 5. Measured capacitance versus frequency with Moore's measurements from [5]. 


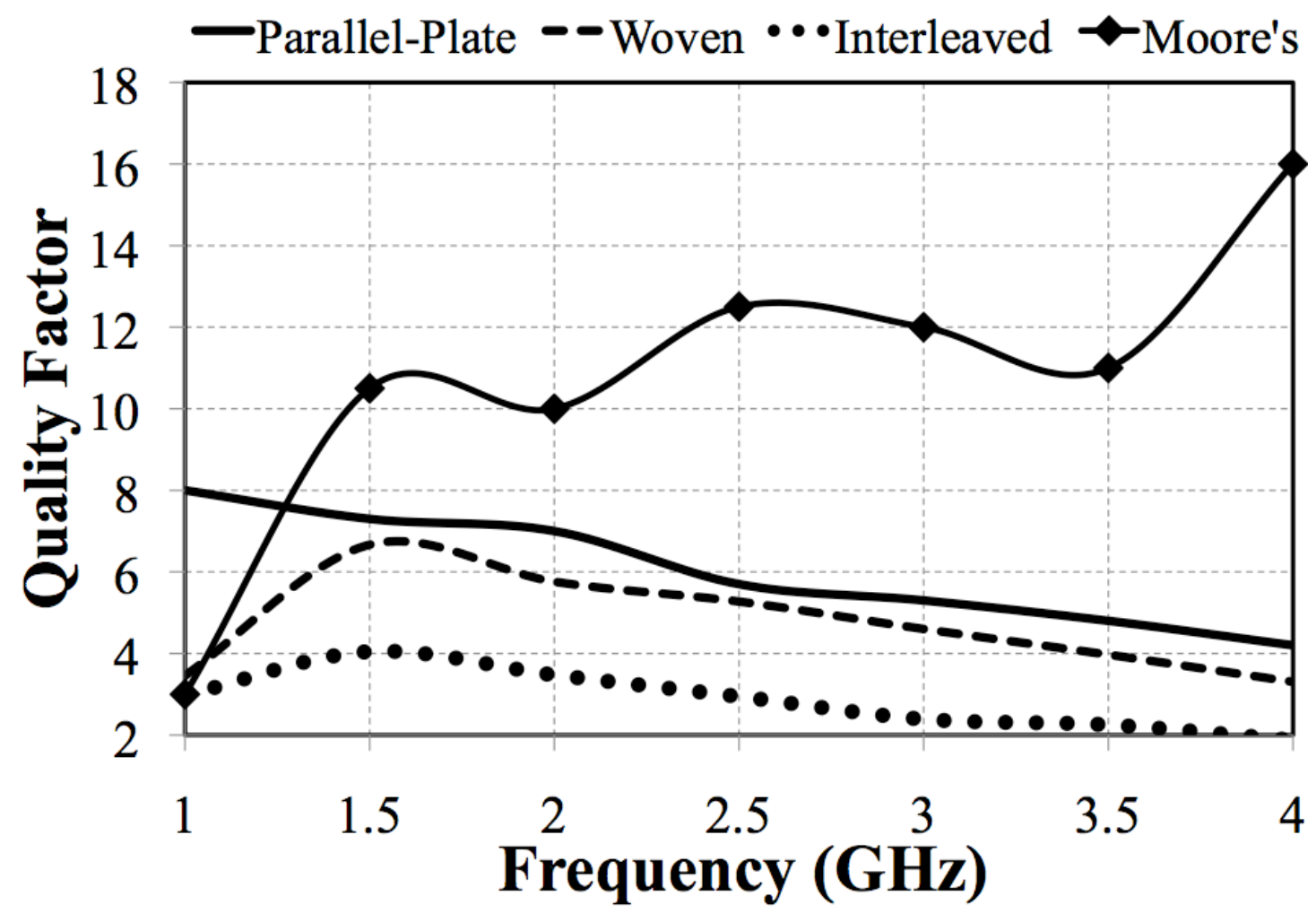

Fig. 6. Measured quality factor versus frequency with Moore's measurements from [5]. 